\title{
Disposable immunosensor using a simple method for oriented antibody immobilization for label-free real-time detection of an oxidative stress biomarker implicated in cancer diseases
}

\author{
Nádia S. Ferreira, M. Goreti F. Sales
}

\begin{abstract}
A B S T R A C T
This work proposes a novel approach for a suitable orientation of antibodies $(\mathrm{Ab})$ on an immunosensing platform, applied here to the determination of 8-hydroxy-2'-deoxyguanosine (80HdG), a biomarker of oxidative stress that has been associated to chronic diseases, such as cancer. The anti-80HdG was bound to an amine modified gold support through its Fc region after activation of its carboxylic functions. Nonoriented approaches of $\mathrm{Ab}$ binding to the platform were tested in parallel, in order to show that the presented methodology favored $\mathrm{Ab} / \mathrm{Ag}$ affinity and immunodetection of the antigen.

The immunosensor design was evaluated by quartz-crystal microbalance with dissipation, atomic force microscopy, electrochemical impedance spectroscopy (EIS) and square-wave voltammetry. EIS was also a suitable technique to follow the analytical behavior of the device against 80HdG. The affinity binding between $80 \mathrm{HdG}$ and the antibody immobilized in the gold modified platform increased the charge transfer resistance across the electrochemical set-up. The observed behavior was linear from 0.02 to $7.0 \mathrm{ng} / \mathrm{mL}$ of $80 \mathrm{HdG}$ concentrations. The interference from glucose, urea and creatinine was found negligible. An attempt of application to synthetic samples was also successfully conducted.

Overall, the presented approach enabled the production of suitably oriented Abs over a gold platform by means of a much simpler process than other oriented-Ab binding approaches described in the literature, as far as we know, and was successful in terms of analytical features and sample application.
\end{abstract}

Keywords:

Immunosensor

Oriented antibody binding

Cancer biomarker

8-Hydroxy-2'-deoxyguanosine

\section{Introduction}

Immunosensors combine an antibody as biological recognition element with a transducer signaling device that follows the binding of the free antigen (Ag) present in the solution under analysis and the antibody $(\mathrm{Ab})$ anchored to a solid support. The transducer converts electrical, optical, mass or heat changes of the solution into a measurable signal (Byrne et al., 2009; D'Orazio, 2003).

A critical step in immunosensor design is the immobilization of the Ab on the solid support (Matharu et al., 2012). An Ab is a large protein composed of hundreds of amino acids, arranged in a tridimensional order that is recognized as a Y shape and where the carboxyl $(-\mathrm{COOH})$ end of the peptide chain is positioned at the lower end of this Y-shape structure. This region is also recognized as Fc. Each Ab binds to a specific part of an $\mathrm{Ag}$ (epitope region), through the two upper-end parts of this $Y$ shape (paratope region) that are amine-terminated. Each $\mathrm{Ab}$ is able to bind two $\mathrm{Ag}$ species because there are two paratope sites in a single structure. This is also feasible when the $\mathrm{Ab}$ is attached to solid-supports, provided that the $\mathrm{Ab}$ is attached through the Fc region, therefore leaving both paratope sites free for Ag binding.

Many approaches for immobilizing antibodies on a solid support lead to random orientation of these proteins all over the modified surface, creating supports of low density binding sites and of decreased binding affinity (Song et al., 2012). These include physical/electrostatic non-covalent adsorption or covalent coupling via amine moieties, yielding surfaces highly covered by antibodies with inactive orientation due to steric blocking of the paratope sites (Alves et al., 2012). Despite resulting in a significant loss of Ab activity, these methods are still commonly used due to their simplicity of execution.

Alternative strategies leading to suitable $\mathrm{Ab}$ orientation have also been presented (Alves et al., 2012; Kausaite-Minkstimiene et al., 2010; Song et al., 2012). The most common approach includes attaching protein $A$ or protein $G$ to the biosensing surface prior to $\mathrm{Ab}$ binding. These proteins bind specifically to the $\mathrm{FC}$ region of the antibodies, providing a suitable orientation of the antibodies all over the surface. However, this additional step upon the surface modification decreases the ordered organization upon 
the biosensing surface, simply because the proteins are also randomly attached to the surface. Other approaches employ the reduction of disulfide bonds between the peptide chains present in the same $A b$, using the resulting thiol side chains as reactive sites for conjugation to gold or maleimide functionalized surfaces (Brogan et al., 2003; Lofas et al., 1995; Vikholm-Lundin, 2005). This approach leads to many inactive $\mathrm{Ab}$ fragments where sidedisulfide bonds may be reduced accidently (Alves et al., 2012). The orientation of these fragments upon the surface is also not effective because in practice it becomes randomly bond to a gold surface, yielding many non-specific regions for protein binding. Other approaches include oxidizing carbohydrate chains that are present on the antibodies, forming reactive aldehyde groups that can bind amine functionalized surfaces (Han et al., 2010). This technique provides a high coupling yield, but the denaturing conditions and oxidative chemicals used can result in a significant loss of $\mathrm{Ab}$ activity. Overall, methods reporting oriented $\mathrm{Ab}$ binding involve complex chemical procedures, requiring many stages of chemical modification that decrease the technical control over the nanostructures and some of these stages may also hinder Ab activity (Alves et al., 2012). This stresses the need for the development of a simple method for site oriented immobilization.

Thus, this work proposes a different and simpler immunosensor design, by activating the carboxylic residues at the Fc site (via carbodiimide reaction) and coupling these directly to an amine functionalized surface. This carboxylic activation process has been employed for decades for protein covalent binding and is expected to be compatible with protein structures, therefore preventing significant loss of activity of $\mathrm{Ab}$. In addition, although it may affect all carboxylic functions in the outer layer of the protein structure, there is a strong probability that most of the antibodies become suitably oriented. Among the 20 amino acids in nature, only aspartic and glutamic acids have carboxylic acid side chains, and these would only be affected if exposed/directed to the outer surface of the 3D structure of the protein.

This concept is here applied to an electrochemical impedance spectroscopy (EIS) transduction, a direct and label-free method for probing protein binding events such as those from $\mathrm{Ab}-\mathrm{Ag}$ interactions (Guan et al., 2001; Holford et al., 2012; Ronkainen et al., 2010). It offers fast, accurate, sensitive, selective and quantitative responses, coupled with portability, low cost and minimal power requirements. EIS is also independent from sample turbidity or optical pathway, widening the range of samples to which it may be applied.

As target analyte, a biomarker of oxidative stress will be employed. Oxidative stress (OS) is the imbalance between oxidantproducing systems and antioxidant defense mechanisms (such as, for example, superoxide dismutase-SOD, and glutathione), resulting in an excessive production of reactive oxygen species (Mancuso et al., 2012; Ziech et al., 2010). This condition is widely recognized as a central feature of many biological processes and diseases, due to their impact on cell injury and death, being involved in aging, neurodegenerative diseases, such as Alzheimer's disease, Parkinson's disease, Huntington's disease, and cancer (Cui et al., 2012; Repine et al., 1997; Zwart et al., 1999). Oxidative adducts derived from DNA (Hwang, 2004), proteins, lipids, and sugars, are widely used to measure OS levels in biological samples (Ogino and Wang, 2007). This includes 8-hydroxy-2'-deoxyguanosine $(8 \mathrm{OHdG})$ which acts as an indicator of DNA oxidation (Ogino and Wang, 2007; Valavanidis et al., 2009; Zwart et al., 1999). Nowadays, the methods used to quantify this biomarker involve complex, expensive and non-portable techniques (Fan et al., 2012; Li et al., 2011; Wang et al., 2011; Zitka et al., 2011), which impair a more effective utilization of OS biomarkers as a predictive tool for degenerative disease installation. Only an immunosensor is presented very recently in literature for $80 \mathrm{HdG}$, but the overall procedure is very complex (Darwish et al., 2013).
Thus, this work describes a novel and simple approach for designing an immunosensor presenting an Ab for 80HdG (anti$80 H d G$ ) suitably oriented over a gold support, here tested for the detection of $80 \mathrm{HdG}$, a cancer biomarker in urine related to nucleic acid oxidation.

\section{Experimental section}

\subsection{Apparatus}

The electrochemical measurements were conducted with a potentiostat/galvanostat from Metrohm Autolab, controlled by NOVA 1.9 software. Atomic force microscopy measurements were made in using tapping mode using a Veeco Metrology Multimode, Nanoscope IVA. Quartz crystal microbalance (QCM) studies were conducted in a QCM E4 from Q-sense, Biolin, with Dissipation monitoring (QCM-D). A DXR ${ }^{\mathrm{TM}}$ Raman Microscope from Thermo Scientific $^{\mathrm{TM}}$ was used for Raman spectrometry with confocal microscopy.

\subsection{Gold supports}

Electrochemical studies used Au-screen printed electrodes (Au-SPEs) purchased from DropSens (DS-C223BT). These had working and counter electrodes of gold and reference electrode and electrical contacts made of silver, interfaced in a switch box from DropSens to enable its potentiostat/galvanostat reading. The gold layer of the electrode was washed with alcohol (70\%) and de-ionized water before any use. All chemical modifications attempted with these electrodes were made only by applying the reacting solution solely on the working electrode.

AFM studies were conducted in Au-glass slides, where the Au layer was deposited by sputtering to ensure its flatness. This gold layer was cleaned by gentile procedures before use, with Piranha solution.

QCM-D studies were conducted with commercial chips from Q-sense, Biolin. These had $14 \mathrm{~mm}$ diameter, $0.3 \mathrm{~mm}$ thickness quartz glass with a flat Au-layer on top and were cleaned with de-ionized water before use.

\subsection{Reagents and solutions}

All chemicals were of analytical grade and water was deionized or ultrapure Milli- $Q$ laboratory grade. Potassium hexacyanoferrate III $\left(\mathrm{K}_{3}\left[\mathrm{Fe}(\mathrm{CN})_{6}\right]\right)$ and potassium hexacyanoferrate II $\left(\mathrm{K}_{4}\left[\mathrm{Fe}(\mathrm{CN})_{6}\right]\right)$ trihydrate were obtained from Riedel-deHaen; bovine serum albumin (BSA), creatinine, urea, $N$-hydroxysuccinimide (NHS), $N$-ethyl- $N$-(3-dimethylaminopropyl) carbodiimide hydrochloride (EDAC), 8-hydroxy-2-deoxyguanosine 98\% (8OHdG), and 8-hydroxyguanosine, GTX (Ab-8OHdG) from Sigma; cysteamine and ethylenediamine from Merck; and dextrose anhydrous from Fisher.

Piranha solution was prepared by mixing in a cabinet $\mathrm{H}_{2} \mathrm{SO}_{4}$ and $\mathrm{H}_{2} \mathrm{O}_{2}$, in 5:1 ratio, using concentrated $\mathrm{H}_{2} \mathrm{SO}_{4}$ solution (95-97\%, Scharlau) and a $30 \% \mathrm{H}_{2} \mathrm{O}_{2}$ (VWR). The $\mathrm{Ab}-8 \mathrm{OHdG}$ solution was used after a $150 \times$ dilution of the commercial product. A $1 \times$ PBS buffer, pH 7.3 (Amresco, E404, Biotechnology grade) was used throughout this work. Synthetic urine was prepared as indicated in Martinez et al., 2007.

\subsection{Antibody immobilization}

The reactions used for $A b$ immobilization are shown in Fig. 1. A solution of cysteamine ( $50 \mathrm{mM})$ was placed on clean Au for $1 \mathrm{~h}$. Only the necessary volume to cover the working electrode area 


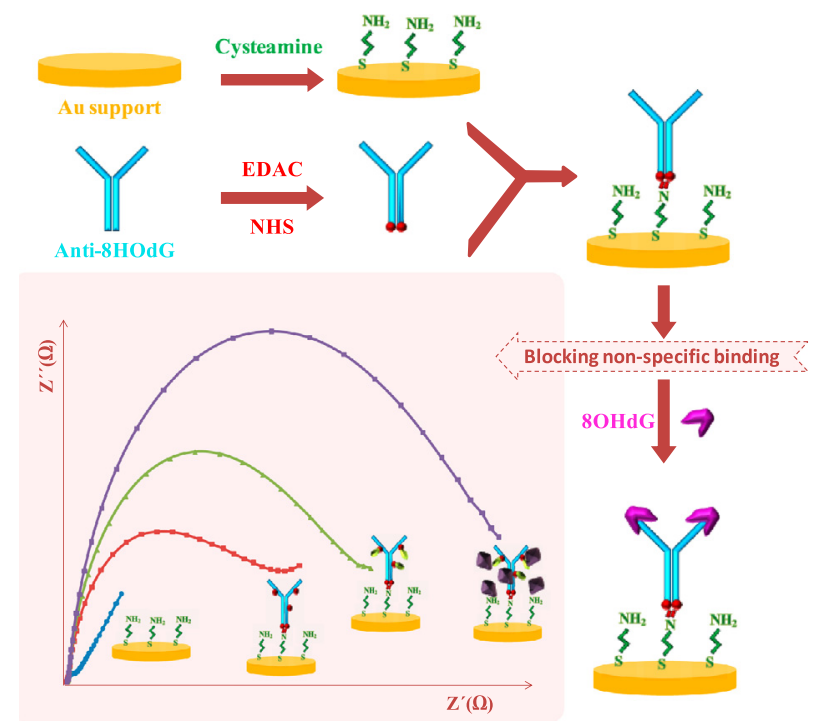

Fig. 1. Design of the immunosensor. TOP: amine modification of gold in parallel with the activation of carboxylic functions of the $\mathrm{Ab}$, and subsequent binding of both materials in a single platform; right: binding the Ag 80HdG to the $\mathrm{Ab}$ in the platform, after blockage of the non-specific response (inset: EIS spectra of $\mathrm{Au} / \mathrm{amine}, \mathrm{Au} / \mathrm{amine} / \mathrm{Ab}, \mathrm{Au} / \mathrm{amine} / \mathrm{Ab}$ with ethylenediamine and $\mathrm{Au} / \mathrm{amine} / \mathrm{Ab}$ with BSA).

was used, in order to prevent the solution from flowing outside the working area (Fig. S1). Before binding the newly formed $\mathrm{Au}-\mathrm{NH}_{2}$ layer, the $\mathrm{Ab}-8 \mathrm{OHdG}$ were modified by reaction with NHS (50 mM) and EDAC ( $25 \mathrm{mM}$ ) prepared in buffer (MES, $50 \mathrm{mM}$, $\mathrm{pH} 6$ ), for $30 \mathrm{~min}$. The modified Ab-8OHdG structures were added after to the $\mathrm{Au}-\mathrm{NH}_{2}$ layer for $1.5 \mathrm{~h}$, at room temperature. Ethylenediamine (20 mM, in PBS) was added next, for $2 \mathrm{~h}$, to deactivate the sites remaining active at the protein structure. BSA $(0.5 \mathrm{mg} / \mathrm{mL}$, in PBS) was added later, for $1 \mathrm{~h}$, at $4{ }^{\circ} \mathrm{C}$, to block the non-specific response of the $\mathrm{Ab}$.

\subsection{Antibody/antigen affinity reaction}

All procedures were made in triplicate. The time given for the affinity reaction between the Ab-80HdG immobilized in the Aulayer and the $\mathrm{Ag}$ (8OHdG, dispersed in the solution), was set to $15 \mathrm{~min}$. The working $8 \mathrm{OHdG}$ solutions had varying concentrations and were prepared in PBS buffer ( $\mathrm{pH} 7.3$ ) by accurate dilution of a stock solution of $20 \mathrm{ng} / \mathrm{mL}$.

\subsection{Electrochemical experiments}

EIS assays were conducted with a redox probe of $\left[\mathrm{Fe}(\mathrm{CN})_{6}\right]^{3-}$ $(5.0 \mathrm{mM})$ and $\left[\mathrm{Fe}(\mathrm{CN})_{6}\right]^{4-}(5.0 \mathrm{mM})$, prepared in PBS buffer, $\mathrm{pH}$ 7.3, after $\mathrm{Ab} / \mathrm{Ag}$ affinity. This was done at a standard potential of $+0.12 \mathrm{~V}$, using a sinusoidal potential perturbation with amplitude of $0.01 \mathrm{~V}$ and a number of frequencies equal to 50 , logarithmically distributed over a frequency range of $0.1-100 \mathrm{kHz}$. The recorded EIS data was fitted to a Randles equivalent circuit, using the Nova Software from Autolab.

Selectivity studies were conducted by competitive assay between $80 H d G$, with a $3 \mathrm{ng} / \mathrm{mL}$ concentration, and urea $(0.5 \mathrm{mg} / \mathrm{mL})$, creatinine $(0.012 \mathrm{mg} / \mathrm{mL})$, and glucose $(1.1 \mathrm{mg} / \mathrm{mL})$, all solutions prepared in PBS buffer, $\mathrm{pH}$ 7.3.

The calibration curves were made for $80 \mathrm{HdG}$ concentrations ranging from 0.02 to $7.0 \mathrm{ng} / \mathrm{mL}$, and followed by both EIS and square-wave voltammetry (SWV) measurements. SWV assays were made by potential scanning from -0.2 to $+0.5 \mathrm{~V}$, at $50 \mathrm{mV} / \mathrm{s}$, for a frequency of $50 \mathrm{~Hz}$ and a step height of $150 \mathrm{mV}$.

\subsection{QCM-D experiments}

QCM-D assays were made after stabilizing the baseline of a cleaned gold chip with PBS carrier, pH 7.3, flowing at $0.50 \mu \mathrm{L} / \mathrm{min}$. Once the equilibrium was reached, the flow was stopped to change this solution by a solution of Ab-80HG (1:10), prepared in the same PBS buffer. Finally, and after reaching again the equilibrium, the flow was once more stopped to introduce a solution of $80 \mathrm{HG}$, $100 \mathrm{ng} / \mathrm{mL}$.

\section{Results and discussion}

\subsection{Antibody/antigen binding}

The ability of the anti-80HdG to bind to its target compound when attached to a solid support of Au was checked by QCM-D studies. Fig. 2 shows the diagrams obtained for frequency and dissipation data versus time.

The $\Delta f$ values decreased significantly after injection of the Ab$80 \mathrm{HdG}$, which confirmed the physical adsorption of the Ab to the Au layer. The saturation was almost reached at 20 min contact. The observed increase in dissipation indicated that the formed film had viscoelastic properties, which is consistent with the random adsorption of the $\mathrm{Ab}$ (of protein nature) on $\mathrm{Au}$.

The Ag was then bound to its Ab layer, leading to a very small increase in the observed frequency under equilibrium. This increase is typically correlated to a mass decrease, which in a first approach seems inconsistent with the binding of 80HdG to the Au/ $\mathrm{Ab}$ layer. However, the molar mass of the $\mathrm{Ag}(283.2 \mathrm{~g} / \mathrm{mol})$ is very small compared to that of the $\mathrm{Ab}$, meaning that this binding stage would not generate a significant mass increase. Since the observed frequency increase is also linked to slight decrease in dissipation, this behaviour may be attributed to the alteration of the protein $(\mathrm{Ab})$ conformation after $80 \mathrm{HdG}$ binding, leading to the extraction of hydration molecules over the $\mathrm{Ab} / \mathrm{Au}$ layer and thus to a subsequent mass loss.

Overall, QCM-D studies confirmed that the Ab is able to bind its Ag in PBS environment and when adsorbed to a solid support, and that the layer of $\mathrm{Ab}$ has a non-rigid structure showing viscoelastic properties.

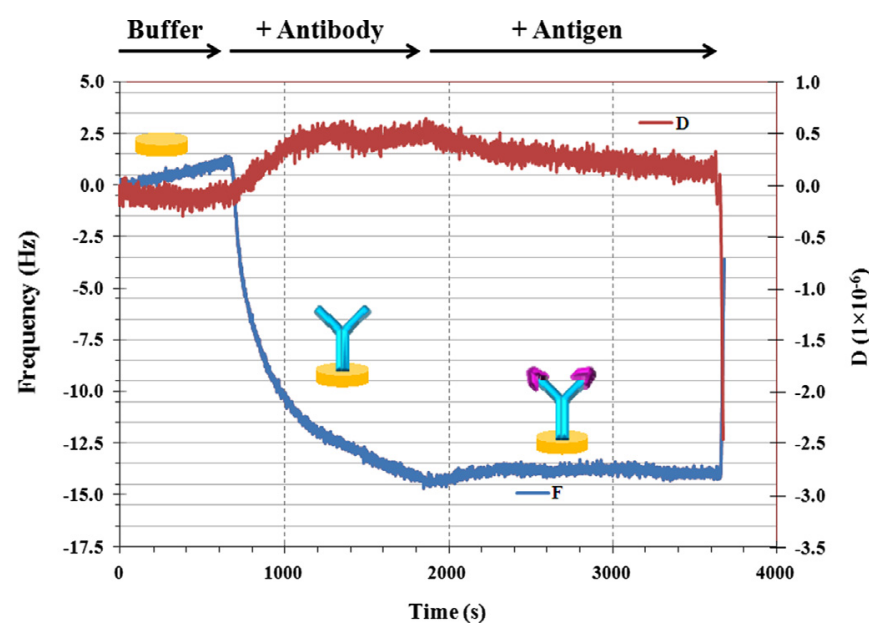

Fig. 2. QCM-D records reporting measurements of frequency (blue, left $Y$ axis) and dissipation (red, right $Y$ axis) against time for the different stages of the reaction (as reported on top). (For interpretation of the references to color in this figure legend, the reader is referred to the web version of this article.) 


\subsection{Immunosensor design}

The two paratope sites in each immobilized $\mathrm{Ab}$ can bind $\mathrm{Ag}$ molecules only if these regions can be easily accessed. For this purpose, it is essential that each Ab bounded to the solid support should be attached through its Fc region. Considering that this region carries carboxylic functions, its activation via carbodiimide reaction would allow its easy and mild covalent binding to an amine layer.

So, the overall design of the immunosensor started by creating an amine layer over the gold support (Fig. 1). This was done by placing cysteamine on Au, a two carbon chain carrying an amine group $\left(-\mathrm{NH}_{2}\right)$ in one end and a thiol function $(-\mathrm{SH})$ in the other end. The contact between Au and - SH leads to the spontaneous formation of a strong Au-sulphur interaction, that is expected to form a closely packed monolayer. In practice, the Au surface becomes a stable amine layer ( $\mathrm{Au} / \mathrm{amine}$ ), that was controlled by EIS studies, presented in the inset of Fig. 1.

The carboxylic functions on the Ab were activated in parallel, in order to enable its reaction with the amine layer under mild conditions. This was done by the well know EDAC/NHS chemistry, where the $-\mathrm{COOH}$ group forms a highly reactive $\mathrm{O}$-acylisourea intermediate that reacts rapidly with NHS to produce a more stable succinimydyl ester intermediate (Jiang et al., 2004). The resulting ester undertakes easy nucleophilic substitution with the amine groups on the $\mathrm{Au} /$ amine layer. The presence of $\mathrm{Ab}$ attached to the amine layer (Au/amine/Ab) was confirmed by a significant increase of the charge-transfer resistance $\left(R_{c t}\right)$ observed in the EIS spectra. The $R_{c t}$ corresponds to the diameter of the semicircle and measures directly the ability of the charge transfer to take place between electrodes and surrounding electrolyte. Thus, the significant increase in the diameter of the semicircle (Fig. 1, inset) showed an increased resistance in the electron transfer kinetics of the redox-probe, accounting the formation of a resistive layer due to the presence of protein material at the sensory surface.
Because this carboxylic activation occurring in the Ab material was not carried out specifically at the $-\mathrm{COOH}$ groups of the Fc region, other carboxylic functions present on the outer surface of the $3 \mathrm{D}$ structure of the $\mathrm{Ab}$ could undergo the same reaction and become activated. In such condition, the target compound or other amine-based compounds in a biological sample could bind to these activated positions, contributing to decrease the selectivity of the immunosensor. So, the carboxylic functions that remained activated after $\mathrm{Ab}$ binding were inactivated by reaction with ethylenediamine (inset in Fig. 1). This reagent is small in size, having a two carbon chain with two end-amine functions, which is expected to increase the effectiveness of the reaction and cause little disturbance upon the 3D arrangement of the $\mathrm{Ab}$.

Being the $\mathrm{Ab}$ a protein of enormous dimensions compared to its target analyte, many of its surface area is able to interact with other substances, especially those of protein nature. This is a proprietary feature of any $A b$ that decreases the selectivity of response in immunosensing. This has been solved in the literature by adding a protein (different than the $\mathrm{Ab}$ or the $\mathrm{Ag}$ ) of low cost, typically BSA, in a high amount. The addition of BSA to the immunosensor under development promoted a significant increase in the $R_{c t}$ value, as may be seen in the inset of Fig. 1. Again, this record accounted the formation of a resistive barrier that caused a significant impedance increase, thus confirming BSA binding to the $\mathrm{Au} / \mathrm{amine} / \mathrm{Ab}$ surface.

\subsection{AFM images}

The AFM images of clean gold and Au/amine/Ab supports with or without Ag may be seen in Fig. 3. The top image shows the typical observation obtained with clean gold. The surface was not exactly flat because the gold support had been reused from a previous experiment.

The presence of the anti-8OHdG on the Au/amine support was evident from the significant change observed in the surface
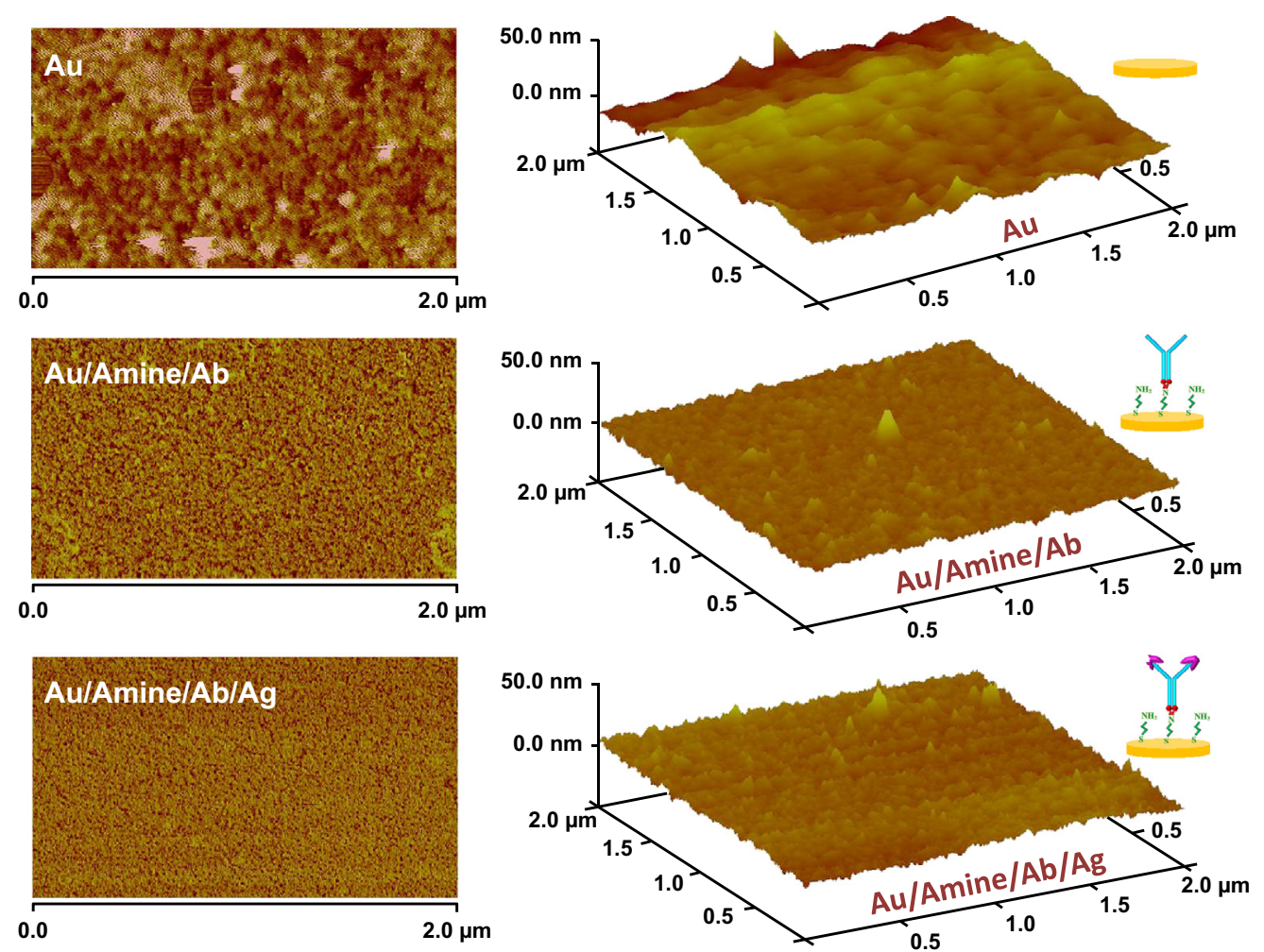

Fig. 3. AFM images in $2 \mathrm{D}$ (left) and 3D (right) views of different materials: Au layer (top), Au/amine/Ab (middle) and Au/amine/Ab with antigen (bottom). 
roughness (Fig. 3, middle). The observed surface was also quite uniform, suggesting that most of the antibodies had a similar orientation after binding, expectedly through the Fc region.

Finally, the addition of Ag also produced a small change in the surface of the material (Fig. 3, bottom). This was more evident in the 2D image. Considering the small size of the Ag, its binding to the $\mathrm{Au} / \mathrm{amine} / \mathrm{Ab}$ support should not give rise to such a significant change at the obtained AFM image. However, this corroborates well with the QCM-D data, regarding the conformational change in the $\mathrm{Ab}$ after $\mathrm{Ag}$ binding.

\subsection{Comparing to non-oriented design}

In order to check if the modification of the Fc region via carboxylic activation was leading to an oriented binding of the $\mathrm{Ab}$ and/or enhancing the analytical features of the biosensor, nonoriented approaches were also established. Two different methods were tested for this purpose: (i) non-specific adsorption of the $\mathrm{Ab}$ to clean Au chips (Au/Ab-adsorbed); (ii) and non-specific adsorption of the $\mathrm{Ab}$ to the $\mathrm{Au} / \mathrm{amine}$ layer (Au/amine/Ab-adsorbed).

The response of such immunosensors against 80HdG is presented in Fig. 4, along with the response of the oriented approach previously described. Overall, the binding events between $80 \mathrm{HdG}$ and anti-80HdG in the immunosensor lead to an increase in $R_{c t}$. According to literature, it is common that the formation of affinity complexes leads to the formation of an insulating layer that retards the interfacial electron transfer kinetics between the redox probe and the electrode, thus increasing the electrontransfer resistance (Ansari et al., 2010).

Comparing the immunosensors designed with oriented and non-oriented approaches, it was clear that the non-controlled $\mathrm{Ab}$ binding versions gave rise to devices with lower reproducibility, because it was difficult to find repeated behaviour in devices produced together. In addition, the spectra presented in Fig. 4 are clear with regard to the narrower and worse linear ranges obtained with non-oriented designs. Immunosensors Au/Ab-adsorbed displayed linear behaviour from 0.4 to $3.0 \mathrm{ng} / \mathrm{mL}$, with a squared correlation coefficient of 0.955 , and immunosensors of Au/amine/ $\mathrm{Ab}$-adsorbed had linear behaviour from 0.020 to $3.0 \mathrm{ng} / \mathrm{mL}$, with a squared correlation coefficient of 0.9405 , and immunosensors of $\mathrm{Au} / \mathrm{amine} / \mathrm{Ab}$ were linear all over the $80 \mathrm{HdG}$ concentration range tested.

\subsection{Main analytical features}

The calibration curves of the oriented immunosensors were evaluated by both EIS and SWV studies, and the main analytical features extracted from the average results of at least three independent assays. The typical records are also shown in Fig. 5.

The EIS calibration was made by plotting $R_{c t}$ values of the EIS spectra against $80 \mathrm{HdG}$ concentration. A typical spectra is shown in Fig. 5A, corresponding to $R_{c t}(\Omega)=685.23 \times[80 \mathrm{HdG}, \mathrm{ng} / \mathrm{mL}]+981.4$,

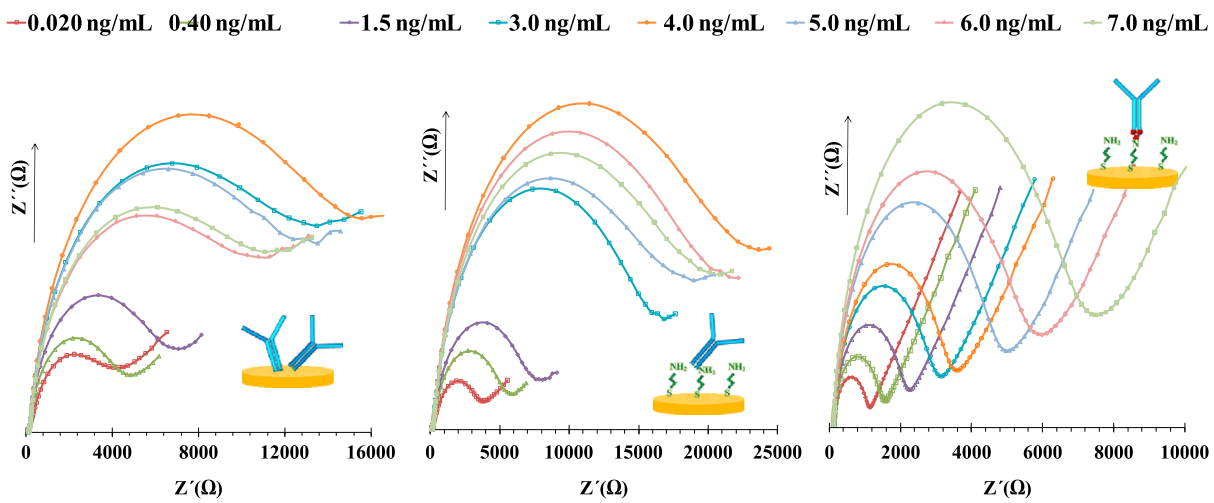

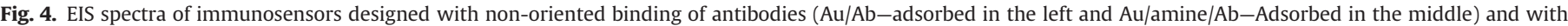
$\mathrm{Ab}$ covalently attached through the Fc activated region (Au/amine/Ab in the right), obtained for different $80 \mathrm{OdG}$ standard concentrations.

$$
\begin{array}{lllll}
- \text { Blank } & -0.02 \mathrm{ng} / \mathrm{mL} & -0.4 \mathrm{ng} / \mathrm{mL} & -1.5 \mathrm{ng} / \mathrm{mL} & -3.0 \mathrm{ng} / \mathrm{mL} \\
-4.0 \mathrm{ng} / \mathrm{mL} & -5.0 \mathrm{ng} / \mathrm{mL} & -6.0 \mathrm{ng} / \mathrm{mL} & -7.0 \mathrm{ng} / \mathrm{mL}
\end{array}
$$

A

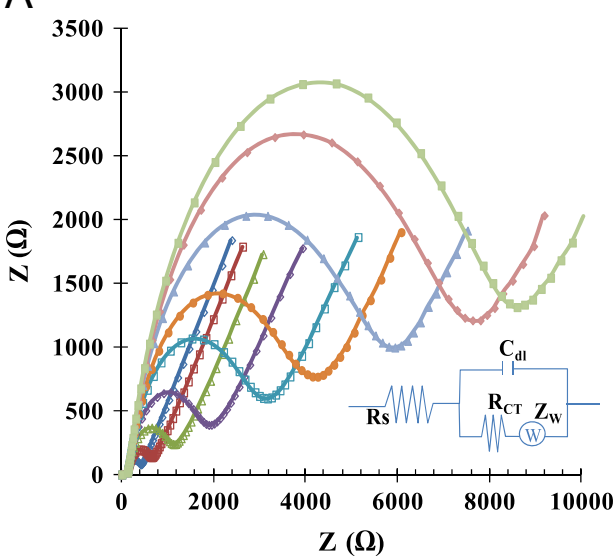

B

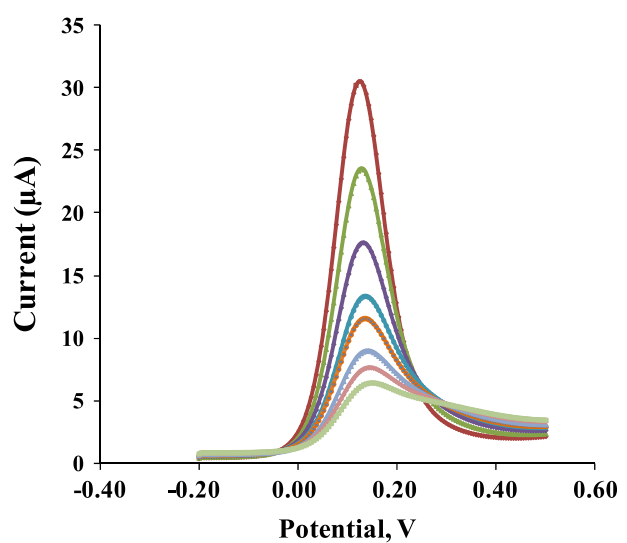

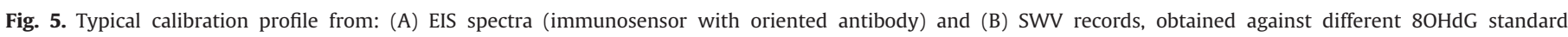

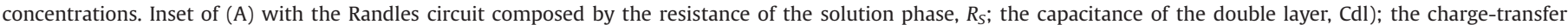
resistance $\left(R_{c t}\right)$; and the Warburg diffusion element $(W)$ from the bulk solution to the electrode interface. 
with a squared correlation coefficient of 0.9949 . This behavior included the concentration values tested, from 0.020 to $7.0 \mathrm{ng} / \mathrm{mL}$, but not all data points. This is however an occasional event as may be seen from the typical calibration observed for other immunosensor devices built-up in parallel (in Fig. S2). The regular 8OHdG levels in urine lye within this range, meaning that the use of this immunosensor may be extended to elevated levels of this cancer biomarker in urine samples. A triplicate assay with different devices showed that this extended linearity range was a reproducible behavior. Squared correlation coefficients were $>0.991$, attributing also good linearity features to the observed response. Absolute slope values were less reproducible from chip to chip calibration. This variability was mostly attributed to variations in the $\mathrm{Ab}$ solutions and in the working Au layer of each SPE chip, used only in a single calibration and being disposed afterwards. In practical terms, this slope variability within different units had no effect on the immunosensor applicability, because the devices were to be applied to samples by the standard addition method (as direct calibration was unfeasible due to the irreversible response of the device).

In SWV studies, the signal measured reflected the decreasing ability of the platform to oxidize the iron species in +2 oxidation state, due to Ag binding and subsequent blocking of the electrontransfer. In general, the blank signal was the highest value of current obtained and this peak started decaying with the increasing concentration of $80 \mathrm{HdG}$. The typical voltammograms so obtained are presented in Fig. 5B. The calibration was expressed as current $(I, \mu A)=-0.2819 \times[80 \mathrm{HdG}, \mathrm{ng} / \mathrm{mL}]+2.9332$, with a squared correlation coefficient of 0.9904 , for concentrations $\geq 1.5 \mathrm{ng} / \mathrm{mL}$ (Fig. S2).

Comparing both electroanalytical approaches, EIS showed an extended linear range against $80 \mathrm{HdG}$ concentration, and was therefore selected for further evaluation and possible application.

\subsection{Antibody surface coverage}

The area of the working electrode covered by Ab was calculated by confocal microscopy coupled to Raman spectrometry. The Raman spectra was recorded with a $532 \mathrm{~nm}$ laser, in the spectral range of $200-3500 \mathrm{~cm}^{-1}$, for $1 \mathrm{~mW}$ power and 2 exposures of $45 \mathrm{~s}$, in combination with a $50 \times$ objective magnification for focus and collection of Raman-scattered light. The confocal hole was set to $25 \mu \mathrm{m}$.

The spectra of Au layer of the SPE had small Raman signal when compared to that of the Au/amine/Ab. The image captured by the video camera of $\mathrm{Au} / \mathrm{amine} / \mathrm{Ab}$ showed intense fluorescence spots that were more or less homogeneously distributed (Fig. S3A). Since organic molecules swamp or obscure the background Raman signal due their intrinsic fluorescence, these spots seem to be indicating the presence of the antibodies and their distribution along the working support of the SPE. The mapping of the Raman signals of Au/amine/Ab surface area may be seen in Fig. S3B. The surface color in Fig. S3C indicated the distribution of Raman intensities of the peak at $1935 \mathrm{~cm}^{-1}$ Raman shift, a signal that was never present in the Au blank layer. Accounting spectra with Raman intensity over $90 \%$, the Ab coverage was $86.7 \%$, thus confirming the successful modification of the Au-SPE with Ab.

\subsection{Selectivity}

The ability of the immunosensor to discriminate $80 \mathrm{HdG}$ from foreign species was tested by adding a fixed concentration of $80 \mathrm{OdG}$ to the immunosensor, followed by the addition of an interfering compound, present in concentrations that are expected to be equivalent to those observed in normal physiological conditions. This study was applied to glucose, urea and creatinine. In general, the observed selectivity will depend mostly on the selectivity/specificity of the $\mathrm{Ab} / \mathrm{Ag}$ reaction and of the non-specific response presented by the immunosensor platform.

The average $R_{c t}$ changes produced by the addition of glucose, urea and creatinine were $8.3 \pm 3.1 \%, 8.6 \pm 1.5 \%$, and $8.1 \pm 1.8 \%$, respectively. Overall, these values show small variability, attributing the devices a good reproducibility in \% levels. The absolute interference level was also low, suggesting that foreign species will not play a significant role at the response of the immunosensor.

\subsection{Application to urine analysis}

Because $\mathrm{Ab} / \mathrm{Ag}$ binding is irreversible, urine samples had to be analyzed by the standard addition method. For this purpose, blank synthetic urine samples with a background level of 80HdG equal to $1.50 \mathrm{ng} / \mathrm{mL}$ were incubated in the sensor. This concentration level is below the normal physiological levels expected for this oxidized metabolite. After this, consecutive additions of standards were made, ranging from 3.0 to $7.0 \mathrm{ng} / \mathrm{mL}$ (the concentration range used for the normal calibration). The sample concentration was calculated after subtracting the blank signal to all standards, plotting the so obtained $R_{c t}$ data and calculating the $X$ intercept for $Y=0$. The concentration level obtained was $1.59 \mathrm{ng} / \mathrm{mL}$, corresponding to a relative error of $5.8 \%$. This result suggested that the developed immunosensor is leading to accurate data. Its simplicity of design suggests the possibility of using it in routine procedures, once the values are validated against real samples.

\section{Conclusions}

A simple Ab binding procedure is presented, with all stages of analytical modification, including analyte binding, being evaluated by several techniques. Ag binding to the immobilized $\mathrm{Ab}$ seems to produce conformation changes in the antibody and affects the charge resistance transfer over the immunosensing platform for a standard redox probe; additional studies are required to conclude such conformational alterations. The successful analytical features presented by EIS studies suggest that a suitable orientation of the Ab over a gold-modified platform was successfully achieved.

Overall, the immunosensor described here is simple, and may allow routine use of other immunosensor devices on-site, offering lower linear response ranges for the 80HdG than the most sensitive commercial kits available. The time and efforts required for the devices setup are significantly reduced, allowing it to be disposed off afterwards without significant costs.

\section{Acknowledgements}

The authors acknowledge Biolin/Paralab for access to the QCM-D equipment and Thermo/Unicam for access to the Raman Microscopy equipment.

\section{Appendix A. Supplementary material}

Supplementary data associated with this article can be found in the online version at http://dx.doi.org/10.1016/j.bios.2013.09.056.

\section{References}

Alves, N.J., Kiziltepe, T., Bilgicer, B., 2012. Langmuir 28, 9640-9648.

Ansari, A.A., Alhoshan, M., Alsalhi, M.S., Aldwayyan, A.S., 2010. Sensors 10 6535-6581.

Brogan, K.L., Wolfe, K.N., Jones, P.A., Schoenfisch, M.H., 2003. Analytica Chimica Acta 496, 73-80. 
Byrne, B., Stack, E., Gilmartin, N., O'Kennedy, R., 2009. Sensors 9, 4407-4445.

Cui, H., Kong, Y., Zhang, H., 2012. Oxidative stress, mitochondrial dysfunction, and aging. Journal of Signal Transduction , http://dx.doi.org/10.1155/2012/646354. (ID 646354, 13 pages).

D'Orazio, P., 2003. Clinica Chimica Acta 334, 41-69.

Darwish, I.A., Wani, T.A., Khalil, N.Y., Aboul-Fadl, T., Kadi, A.A., Al-Majed, A.R.A. 2013. Analytical Methods 5, 1502-1509.

Fan, R.F., Wang, D.L., Ramage, R., She, J.W., 2012. Chemical Research in Toxicology 25, 491-499.

Guan, J.G., Miao, Y.Q., Zhang, Q.J., 2001. Journal of Bioscience and Bioengineering 97. 219-226.

Han, H.J., Kannan, R.M., Wang, S., Mao, G., Kusanovic, J.P., Romero, R., 2010 Advanced Functional Materials 20, 409-421.

Holford, T.R.J., Davis, F., Higson, S.P.J., 2012. Biosensors and Bioelectronics 34, 12-24.

Hwang, E.S., 2004. Food Science and Biotechnology 13, 253-261.

Jiang, K., Schadler, L.S., Siegel, R.W., Zhang, X., Zhang, H., Terrone, M., 2004. Journal of Materials Chemistry 14, 37-39.

Kausaite-Minkstimiene, A., Ramanaviciene, A., Kirlyte, J., Ramanavicius, A., 2010 Analytical Chemistry 82, 6401-6408.

Li, M.J., Zhang, J.B., Li, W.L., Chu, Q.C., Ye, J.N., 2011. Journal of Chromatography B $879,3818-3822$.

Lofas, S., Johnsson, B., Edstrom, A., Hansson, A., Lindquist, G., Hillgren, R., Stigh, L. 1995. Biosensors and Bioelectronics 10, 813-822.

Martinez, A.W., Phillips, S.T., Butte, M.J., Whitesides, G.M., 2007. Angewandte Chemie International Edition 46, 1318-1320.
Mancuso, M., Bonanni, E., LoGerfo, A., Orsucci, D., Maestri, M., Chico, L., DiCoscio, E., Fabbrini, M., Sicilliano, G., Murri, L., 2012. Sleep Medicine 13, 632-636.

Matharu, Z., Bandodkar, A.J., Gupta, V., Malhotra, B.D., 2012. Chemical Society Reviews 41, 1363-1402.

Ogino, K., Wang, D., 2007. Acta Medica Okayama 61, 181-189.

Repine, J., Bast, A., Lankhorst, I., 1997. American Journal of Respiratory and Critical Care Medicine 156, 341-357.

Ronkainen, N.J., Halsall, H.B., Heineman, W.R., 2010. Chemical Society Reviews 39, 1747-1763.

Song, H.Y., Zhou, X., Hobley, J., Su, X., 2012. Langmuir 28, 997-1004.

Valavanidis, A., Vlachogianni, T., Fiotakis, C., 2009. Journal of Environmental Science and Health, Part C: Environmental Carcinogenesis \& Ecotoxicology Reviews 27. 120-139.

Vikholm-Lundin, I., 2005. Langmuir 21, 6473-6477.

Wang, C.J., Yang, N.H., Chang, C.C., Liou, S.H., Lee, H.L., 2011. Journal of Chromatography 879, 3538-3543.

Ziech, D., Franco, R., Georgakilas, A., Georgakila, S., Malamou-Mitsi, V., Schoneveld, O., Pappa, A., Panayiotidis, M., 2010. Chemico-Biological Interactions 188, 334-339.

Zitka, O., Krizkova, S., Krejcova, L., Hynek, D., Gumulec, J., Masarik, M., Sochor, J., Adam, V., Hubalek, J., Trnkova, L., Kizek, R., 2011. Electrophoresis 32, 3207-3220.

Zwart, L., Meerman, J., Commandeur, J., Vermeulen, N., 1999. Free Radical Biology and Medicine 26, 202-226. 